Research Article

\title{
Performance Ranking Method Based on Superefficiency with Directional Distance Function in DEA
}

\author{
Jing Tang $\mathbb{D},{ }^{1,2}$ Jianzhong Liu, ${ }^{1}$ Jianghua Chen, ${ }^{1}$ and Fangqing Wei $\mathbb{i D}^{2}$ \\ ${ }^{1}$ School of Economics and Management, Hefei University, Hefei, Anhui 230601, China \\ ${ }^{2}$ School of Management, University of Science and Technology of China, Hefei, Anhui 230026, China \\ Correspondence should be addressed to Fangqing Wei; wfq89072@mail.ustc.edu.cn
}

Received 20 November 2019; Revised 10 January 2020; Accepted 16 January 2020; Published 18 February 2020

Academic Editor: Sri Sridharan

Copyright (c) 2020 Jing Tang et al. This is an open access article distributed under the Creative Commons Attribution License, which permits unrestricted use, distribution, and reproduction in any medium, provided the original work is properly cited.

In data envelopment analysis (DEA) methodology, superefficiency models eliminate the DMU to be evaluated from the production possibility set (PPS) to investigate whether its performance is superefficient. However, the infeasibility has been found in the superefficiency models when variable return-to-scale (VRS) technology is assumed. In recent developments, directional distance functions (DDF) are introduced into VRS superefficiency models to address the infeasibility, and the obtained efficiency scores from the DDF-based VRS superefficiency measure are used to rank all DMUs. In this study, we discuss conditions on selecting some proper reference bundles for feasible DDF and suggest a new DDF-based VRS superefficiency measure, which is unit-invariant and does not need to specify additional parameters. Two example illustrations are evaluated to demonstrate the feasibility and usefulness of our proposed DDF-based VRS superefficiency ranking method.

\section{Introduction}

Data envelopment analysis (DEA) is one of the most useful management tools for efficiency evaluation and ranking decision-making units (DMUs). First proposed by Charnes et al. [1], DEA has been widely used in many areas, such as education, transportation, banking, agriculture, and among others [2]. One limitation in conventional DEA models (e.g., CCR and BCC) is that they cannot fully rank those efficient DMUs but simply identify them as CCR or BCC efficient with the same efficiency scores [3]. In other words, there exist ties for efficient DMUs when we are ranking DMUs with the CCR or BCC efficiency scores.

To improve the discriminating ability and obtain a full ranking of all DMUs, especially the efficient ones, a number of researchers put forward different methods, such as superefficiency approach, cross-efficiency measurement, and common weight method [4]. As an effective technique for fully ranking DMUs, a superefficiency approach introduced by Andersen and Petersen [5] has attracted significant attention of many scholars. The superefficiency scores from the model can be greater than one for CCR and BCC efficient which are regarded as the evaluated DMUs whether being superefficient. These superefficiency scores are then used to rank the DMUs and thereby eliminate some (but not all for some cases) of the ties that occur for efficient DMUs. However, there exist infeasible solutions in the conventional superefficiency model for certain DMUs when the variable return-to-scale (VRS) technology is assumed [6]; such infeasibility issue restricts usefulness of the DEA superefficiency model [7]. To address the infeasibility issue, several modified superefficiency approaches have been developed, e.g., scaling up/down the observed input/output values for evaluated DMUs [8], incorporating efficient projections of inefficient DMUs to form a new dataset (e.g., $[9,10]$ moving both inputs and outputs of an efficient but infeasible DMU to the frontier which is formed by the rest of DMUs [11]).

In recent years, directional distance function (DDF) [12] attracts the interest of scholars since such method takes the input extraction and output expansion into account simultaneously in the performance evaluation applications and modellings [13]. As a result, it may yield more comprehensive ranking results than that of inputoriented DEA models or output-oriented DEA models. 
Accordingly, an alternative procedure proposed by Ray [14] is to use the DDF and the resulting Nerlove-Luenberger $(\mathrm{N}-\mathrm{L})$ superefficiency measure under VRS technology assumption. Ray [14] suggested that the NL superefficiency scores obtained from the direction distance function are unique and easily interpreted. Meanwhile, Ray [14] admitted the N-L superefficiency model is feasible in most cases but two exceptions are left: (1) the obtained N-L superefficiency that could be less than -1 yields the negative output improvement target, and (2) the model becomes infeasible when the evaluated DMU has more than one zero input value, whereas that input values of all other DMUs are positive. To deal with the above two exceptions, Chen et al. [7] specified a directional vector in the DDF and obtained a modified DDF-based VRS superefficiency model in which two positive parameters need to be predetermined by a procedure with a set of complex inequalities. But the limitation of the negative output target may exist in Chen et al. [7]'s model as pointed by Lin and Chen [15]. Using the directional vectors defined by Portela et al. [16], Hadi-Vencheh and Esmaeilzadeh. [17] proposed two new DDF-based superefficiency models, namely, super RDM + model and super RDM-model. However, the infeasible issues occurred in both models when the evaluated DMU has the minimum input or the maximum output among all DMUs. Lin and Chen [15] proposed a modified superefficiency model with an appropriate reference bundle in the DDF, which is always feasible. Unfortunately, their model is a purely mathematical concern for well-defined solutions for all DMUs. Later, Lin and Chen [15] gave another DDF-based superefficiency model in the presence of the negative input and output value. But their proposed directional vectors depend on a parameter, which leads to sensitivity issues in the evaluation results [18].

In this paper, to address infeasibility issue in conventional DDF-based VRS superefficiency models, we discuss conditions on choosing the proper reference bundle in the DDF to make DDF-based superefficiency always feasible under VRS. That is, our developed modified superefficiency model has generality which can be used to help the decision maker select appropriate directional vectors along which the efficient frontier is improved. In addition, the new model is independent of preassigned parameters thus eliminating the sensitivity problems that occurred in the evaluation results, and it also embeds the unit-invariant property which means the superefficiency measure is independent of the input and output units. To sum up, this study provides the generality conditions on selecting appropriate directional vectors to tackle the infeasibility issues appeared in both superefficiency models (VRS and $\mathrm{N}-\mathrm{L}$, respectively) introduced by Andersen and Petersen [5] and Ray [14].

The rest of the paper is organized as follows. Section 2 discusses the infeasibility in the DDF-based VRS superefficiency model and proposes our new model. The method is applied to one application of airlines companies for demonstration in Section 3. Section 4 concludes the study.

\section{The VRS Superefficiency with Directional Distance Function}

There are $n$ DMUs with the input and output matrices $X=$ $\left(x_{i j}\right) \in \Re^{m \times n}$ and $Y=\left(y_{r j}\right) \in \mathfrak{R}^{s \times n}$, respectively. For any evaluated $\operatorname{DMU}_{d}(d=1, \cdots, n)$, the production possibility set $T_{d}$ for superefficiency under variable return-to-scale technology assumption [19] is defined as

$$
T_{d}=\left\{\begin{array}{l|l}
\left(x_{i}, y_{r}\right) & \begin{array}{l}
\sum_{j=1, j \neq d}^{n} \lambda_{j} x_{i j} \leq x_{i}, i=1, \cdots, m, \\
\sum_{j=1, j \neq d}^{n} \lambda_{j} y_{r j} \geq y_{r}, r=1, \cdots, s, \\
\sum_{j=1, j \neq d}^{n} \lambda_{j}=1, \\
\lambda_{j} \geq 0, j=1, \cdots, n, j \neq d .
\end{array}
\end{array}\right\}
$$

Considering $\mathrm{DMU}_{d}\left(x_{i d}, y_{r d}\right)$ and the directional vector $\left(g_{x}, g_{y}\right),[12,20]$ define a directional distance function based on the production possibility set $T_{d}$ as follows:

$$
\vec{D}_{T_{d}}\left(x_{i d}, y_{r d} ; g_{x}, g_{y}\right)=\max \beta:\left(x_{i d}+\beta g_{x}, y_{r d}+\beta g_{y}\right) \in T_{d} \text {, }
$$

where $\left(g_{x}, g_{y}\right)$ is a nonzero vector in $\mathfrak{R}^{m} \times \mathfrak{R}^{s}$ and the selection of the directional vectors is arbitrarily (e.g., $[7,14])$. Chambers et al. [12] utilized the observed input and output values $\left(-x_{i d}, y_{r d}\right)$ as $\left(g_{x}, g_{y}\right)$, and the DDF becomes

$$
\vec{D}_{T_{d}}\left(x_{i d}, y_{r d}\right)=\max \beta:\left(x_{i d}-\beta x_{i d}, y_{r d}+\beta y_{r d}\right) \in T_{d} \text {. }
$$

Thus, the VRS N-L superefficiency model for calculating $\mathrm{DMU}_{d}(d=1, \cdots, n)$ is

$$
\begin{array}{ll}
\max & \beta \\
\text { s.t. } & \sum_{j=1, j \neq d}^{n} \lambda_{j} x_{i j} \leq(1-\beta) x_{i d}, i=1, \cdots, m, \\
& \sum_{j=1, j \neq d}^{n} \lambda_{j} y_{r j} \geq(1+\beta) y_{r d}, r=1, \cdots, s, \\
& \sum_{j=1, j \neq d}^{n} \lambda_{j}=1, \lambda_{j} \geq 0, j=1, \cdots, n, j \neq d .
\end{array}
$$

In model (4), the observed inputs and outputs can be scaled by the proportion $\beta$ simultaneously along the direction designed by the directional vector $\left(-x_{i d}, y_{r d}\right)$. The optimal value $\beta$ in (4) is the N-L measure of technical inefficiency of the DMU under evaluation, and the efficiency score equals $(1-\beta)$ [14]. For the efficient $\mathrm{DMU}_{d}(d=1, \cdots, n)$, the optimal value $\beta<0$ means it should scale down its observed outputs while scale up its observed inputs by the same proportion to obtain an available input-output mix in the new production possibility set $T_{d}$ [14]. The DMU with a smaller value of $\beta$ means a higher N-L superefficiency score.

Ray [14] claims that the N-L superefficiency model is feasible in most cases except two cases: first, if the optimal 
value $\beta$ in model (4) is less than -1 , then the output bundle $\left((1+\beta) y_{k}\right)$ is rendered negative [14]. Second, the model is infeasible if at least one element of the input value of the evaluated DMU is zero and all other DMUs in the reference set use the strictly positive value of that input [14]. For any input $x_{i_{o} d}=0,\left(i_{o}=1, \cdots, m\right), x_{i_{j}}>0,\left(i_{o}=1, \cdots, m, j \neq d\right)$ and under the constraints $\sum_{j=1, j \neq d}^{n} \lambda_{j}=1, \lambda_{j} \geq 0$, $(j=1, \cdots, n, j \neq d)$, the first constraint set in model (4) failed and the infeasibility is caused in model (4).

Next, we will discuss the feasible conditions on choosing the directional vectors in VRS superefficiency with a general DDF form. Below is the VRS superefficiency with a general DDF form:

$$
\begin{array}{ll}
\max & \beta \\
\text { s.t. } & \sum_{j=1, j \neq k}^{n} \lambda_{j} x_{i j} \leq x_{i k}-\beta g_{x i}, i=1, \cdots, m, \\
& \sum_{j=1, j \neq k}^{n} \lambda_{j} y_{r j} \geq y_{r k}+\beta g_{y r}, r=1, \cdots, s, \\
& \sum_{j=1, j \neq k}^{n} \lambda_{j}=1, \lambda_{j} \geq 0, j=1, \cdots, n, j \neq k .
\end{array}
$$

For the efficient DMU, $\beta$ with a negative value means it should scale down its observed outputs while scale up its observed inputs to obtain an attainable projection. To ensure that model (5) always has feasible solutions and the outputs have a positive projection, the following inequalities should be satisfied:

$$
\left\{\begin{array}{l}
x_{i k}-\beta g_{x i} \geq 0 \\
\sum_{j=1, j \neq k}^{n} \lambda_{j} x_{i j} \leq x_{i k}-\beta g_{x i}, i=1, \cdots, m \\
y_{r k}+\beta g_{y r} \geq 0 \\
\sum_{j=1, j \neq k}^{n} \lambda_{j} y_{r j} \geq y_{r k}+\beta g_{y r}, r=1, \cdots, s .
\end{array}\right.
$$

Firstly, each value of $g_{x i}, g_{y r}(i=1, \cdots, m, r=1, \cdots s)$ is not equal to zero. Then, formula (6) can be reduced into the following equation:

$$
\left\{\begin{array}{l}
\beta \leq \frac{x_{i k}-\sum_{j=1, j \neq k}^{n} \lambda_{j} x_{i j}}{g_{x i}}, \quad i=1, \cdots, m, \\
-\frac{y_{r k}}{g_{y r}} \leq \beta \leq \frac{\sum_{j=1, j \neq k}^{n} \lambda_{j} y_{r j}-y_{r k}}{g_{y r}}, \quad r=1, \cdots, s .
\end{array}\right.
$$

To ensure that there exist feasible solutions in (7), we obtain following conditions, i.e.,

$$
\max _{r=1, \cdots, s}\left(-\frac{y_{r k}}{g_{y r}}\right) \leq \min _{i=1, \cdots, m}\left(\frac{x_{i k}-\sum_{j=1, j \neq k}^{n} \lambda_{j} x_{i j}}{g_{x i}}\right),
$$

which is rewritten as

$$
-\min _{r=1, \cdots, s}\left(\frac{y_{r k}}{g_{y r}}\right) \leq \min _{i=1, \cdots, m}\left(\frac{x_{i k}-\sum_{j=1, j \neq k}^{n} \lambda_{j} x_{i j}}{g_{x i}}\right) .
$$

Secondly, we consider the two special cases when some elements of $g_{x i}, g_{y r}(i=1, \cdots, m, r=1, \cdots s)$ equal to zero. On the one hand, if $g_{x i_{o}}=0,\left(i_{o}=1, \cdots, m\right)$ and the $i_{o}$-th input of $\mathrm{DMU}_{k}$ equals to zero, but other DMUs has positive values of that input, in other words, $x_{i_{0} k}=0, x_{i_{o}}>0,(k, j=1, \cdots, n, k \neq j)$, then the first constraint of model (5) is not satisfied. On the other hand, if $g_{y r_{o}}=0,\left(r_{o}=1, \cdots, s\right)$ and $y_{r_{o} k}=\max _{j}\left\{y_{r_{o} j}\right\}$, then model (5) fails in the second constraint. Thus, to ensure the VRS superefficiency DDF model (5) always has feasible solutions, the inequality (9) and the following two specify conditions should be satisfied simultaneously:

(a) If $x_{i_{o} j}>0$, then $x_{i_{o} k}=0$ and $g_{x i_{o}}=0$ cannot exist simultaneously at any time, $i_{o}=1, \cdots, m \cdot k, j=1, \cdots, n, k \neq j$.

(b) $y_{r_{o} k}=\max _{j}\left\{y_{r_{o} j}\right\}$ and $g_{y r_{o}}=0$ cannot exist simultaneously at any time, $r_{o}=1, \cdots, s$.

Theorem 1. Given a directional vector $\left(g_{x}, g_{y}\right)$, if inequality (9) and conditions (a) and (b) are satisfied simultaneously, then the corresponding VRS superefficiency DDF model (5) always has feasible solutions. Otherwise, infeasible solutions would be obtained by model (5).

It is not difficult to examine that the proposed directional vector $\left(-x_{i k}-\max _{j}\left\{x_{i j}\right\}, y_{r k}\right)$ in Lin and Chen [15] always satisfies inequality (9) and conditions (a) and (b), while the directional vectors $\left(-x_{i k}, y_{r k}\right)$ in Ray [14] and $\left(-x_{i o}+\min _{j}\left\{x_{i j}\right\}, \max _{j}\left\{y_{r j}\right\}-y_{r k}\right)$ in Portela et al. [16] fail. As a result, their methods cannot eliminate the infeasibility issues in VRS superefficiency models according to Theorem 1.

Next, we develop an alternative specific directional vector which is different from Chen et al. [7] and Lin and Chen [15] to illustrate the other directions are also feasible in the VRS superefficiency DDF model (5) if inequality (9) and above conditions (a) and (b) are satisfied as Theorem 1. Thus, the decision makers could also select other directional vectors depending on his preference and management level or other external conditions, which are not restricted to the directional vector mentioned in Lin and Chen [15]. Our developed directional vector is $\left(-\max _{j}\left\{x_{i j}\right\}+\min _{j}\left\{x_{i j}\right\}, y_{r k}\right)$ for $\left(g_{x}, g_{y}\right)$, and the DDF becomes

$$
\begin{aligned}
\vec{D}_{T}\left(x_{i k}, y_{r k}\right)= & \max \beta:\left(x_{i k}-\beta\left(\max _{j}\left\{x_{i j}\right\}\right.\right. \\
& \left.\left.-\min _{j}\left\{x_{i j}\right\}\right), y_{r k}+\beta y_{r k}\right) \in T .
\end{aligned}
$$


Compared to Lin and Chen [15], here we set a common input direction by its range, which is defined as its maximum observed minus its minimum observed value [21]. Our VRS superefficiency model with the DDF in (10) to measure $\mathrm{DMU}_{k}(k=1, \cdots, n)$ is

$\max \beta$

$$
\begin{array}{ll}
\text { s.t. } & \sum_{j=1, j \neq k}^{n} \lambda_{j} x_{i j} \leq x_{i k}-\beta\left(\max _{j}\left\{x_{i j}\right\}-\min _{j}\left\{x_{i j}\right\}\right), i=1, \cdots, m \\
& \sum_{j=1, j \neq k}^{n} \lambda_{j} y_{r j} \geq y_{r k}+\beta y_{r k}, r=1, \cdots, s \\
& \sum_{j=1, j \neq k}^{n} \lambda_{j}=1, \lambda_{j} \geq 0, j=1, \cdots, n, j \neq k .
\end{array}
$$

Similar to Ray [14], for efficient DMUs, $\beta$ with a negative value means the observed inputs/outputs should be scaled up/down, respectively, to obtain an attainable projection. Theorem 2 shows that the infeasibility issue cannot happen in any case in our developed DDF-based VRS superefficiency methodology.

Theorem 2. Model (11) with the directional vector $\left(-\max _{j}\left\{x_{i j}\right\}+\min _{j}\left\{x_{i j}\right\}, y_{r k}\right)$ is always feasible.

Proof. Firstly, we prove model (11) satisfies the specific conditions (a) and (b). If the value of the $i_{o}$-th input of all DMUs equal to zero, this $i_{o}$-th input will not exist. If $i_{o}$-th input's value of all DMUs are the same, there will be $\max _{j}\left\{x_{i_{o} j}\right\}=\min _{j}\left\{x_{i_{o} j}\right\}(\neq 0),\left(i_{o}=1, \cdots, m, j=1, \cdots, n\right)$, thus $\quad x_{i_{o} k}>0,\left(i_{o}=1, \cdots, m, k=1, \cdots, n,\right)$ but $g_{x i_{o}}=0,\left(i_{o}=1, \cdots, m\right)$. On the other hand, $\max _{j}\left\{x_{i_{j}}\right\} \neq \min _{j}\left\{x_{i_{o}}\right\},\left(i_{o}=1, \cdots, m, j=1, \cdots, n\right)$, then $g_{x i_{o}}>0,\left(i_{o}=1, \cdots, m\right)$, condition (a) is satisfied. It is impossible that the value of the $r_{o}$-th output of all DMUs is equal to zero. If $y_{r_{o} k}=0,\left(r_{o}=1, \cdots, s, k=1, \cdots, n\right)$, at least one of the other DMUs has the positive value of this output value, that is, $y_{r_{o} j}>0,\left(r_{o}=1, \cdots, s, j=1, \cdots, n, j \neq k\right)$ and $y_{r_{o} k}<\max _{j}\left\{y_{r_{o} j}\right\}$, then condition (b) is satisfied.

Secondly, we prove model (11) satisfies the general inequality (9) under the assumption that $g_{x}>0$ and $g_{y}>0$. In model (11), $g_{x i}=\max _{j}\left\{x_{i j}\right\}-\min _{j}\left\{x_{i j}\right\}, g_{y r}=y_{r k}$ and the left hand of inequality (9) equals to $-\min \left(y_{r k} / g_{y r}\right)=-\min \left(y_{r k} / y_{r k}\right)=-1$, then we just prove that

$$
\min _{i=1, \cdots, m}\left(\frac{x_{i k}-\sum_{j=1, j \neq k}^{n} \lambda_{j} x_{i j}}{\max _{j}\left\{x_{i j}\right\}-\min _{j}\left\{x_{i j}\right\}}\right) \geq-1 .
$$

Considering the left hand of inequality of (12) because of $\sum_{j=1, j \neq k}^{n} \lambda_{j} x_{i j} \leq \max _{j}\left\{x_{i j}\right\}$, we can obtain

$$
\frac{x_{i k}-\sum_{j=1, j \neq k}^{n} \lambda_{j} x_{i j}}{\max _{j}\left\{x_{i j}\right\}-\min _{j}\left\{x_{i j}\right\}} \geq \frac{x_{i k}-\max _{j}\left\{x_{i j}\right\}}{\max _{j}\left\{x_{i j}\right\}-\min _{j}\left\{x_{i j}\right\}} .
$$

As $x_{i k} \geq \min _{j}\left\{x_{i j}\right\},(i=1, \cdots, m)$, the right hand of the inequality (12a) becomes $\left(x_{i k}-\max _{j}\left\{x_{i j}\right\} / \max _{j}\right.$ $\left.\left\{x_{i j}\right\}-\min _{j}\left\{x_{i j}\right\}\right)=\left(-\max _{j}\left\{x_{i j}\right\}+\min _{j}\left\{x_{i j}\right\}-\min _{j}\left\{x_{i j}\right\}+\right.$

$\left.x_{i k} / \max _{j}\left\{x_{i j}\right\}-\min _{j}\left\{x_{i j}\right\}\right)=-1+\quad\left(x_{i k}-\min _{j}\left\{x_{i j}\right\} / \max _{j}\right.$

$\left.\left\{x_{i j}\right\}-\min _{j}\left\{x_{i j}\right\}\right) \geq-1$. Then, we obtain

$$
\frac{x_{i k}-\sum_{j=1, j \neq k}^{n} \lambda_{j} x_{i j}}{\max _{j}\left\{x_{i j}\right\}-\min _{j}\left\{x_{i j}\right\}} \geq-1 \text {. }
$$

Thus model (11) satisfies the general inequality (9) under the assumption that $g_{x}>0$ and $g_{y}>0$. In other words, the directional vector $\left(-\max _{j}\left\{x_{i j}\right\}+\min _{j}\left\{x_{i j}\right\}, y_{r k}\right)$ is also proper to make the VRS superefficiency DDF model feasible.

Lovell and Pastor [22] suggested that efficiency measurement should be independent of the units of the measured input and output, which is known as "unit-invariant" or a general mathematical property known as "dimensionless" [22]. Theorem 3 shows our developed model (11) embeds this useful property.

Theorem 3. Model (11) is unit-invariant which does not depend on the units of measurement of the input and output dataset.

Proof. We make all the input and output of the $\mathrm{DMU}_{k}(k=$ $1, \cdots, n) \quad$ scale by $l_{i}\left(l_{i}>0, i=1, \cdots, m\right) \quad$ and $t_{r}\left(t_{r}>0, r=1, \cdots, s\right)$, respectively. Thus, we can obtain

$$
\begin{aligned}
& x_{i j}^{\prime}=l_{i} x_{i j},(i=1, \cdots, m), \\
& y_{r j}^{\prime}=t_{r} y_{r j},(r=1, \cdots, s) .
\end{aligned}
$$

Then, model (11) becomes

$$
\begin{array}{ll}
\max & \beta \\
\text { s.t. } & \sum_{j=1, j \neq k}^{n} \lambda_{j} x_{i j}^{\prime} \leq x_{i k}^{\prime}-\beta\left(\max _{j}\left\{x_{i j}^{\prime}\right\}-\min _{j}\left\{x_{i j}^{\prime}\right\}\right), i=1, \cdots, m \\
& \sum_{j=1, j \neq k}^{n} \lambda_{j} y_{r j}^{\prime} \geq y_{r k}^{\prime}+\beta y_{r k}^{\prime}, r=1, \cdots, s \\
& \sum_{j=1, j \neq k}^{n} \lambda_{j}=1, \lambda_{j} \geq 0, j=1, \cdots, n, j \neq k .
\end{array}
$$

Model (14) is equivalent to model (11), thus model (11) is also unit-invariant.

Next, we take a numerical example from Chen et al. [7] to compare the traditional VRS superefficiency model, N-L model, and Model (11). Table 1 presents the data for five DMUs with two inputs and one output as well as superefficiency results. Here, the superefficiency of $\operatorname{DMU}_{k}(k=$ $1, \cdots, n)$ equals $(1-\beta)$.

In Table 1, column 5 shows the superefficiency scores obtained from the conventional input-oriented VRS superefficiency model. DMUs B and D are inefficient, while DMUs $\mathrm{A}, \mathrm{C}$, and $\mathrm{E}$ are efficient. For $\mathrm{DMU}_{C}, x_{2 C}=0$, nevertheless, $\quad x_{2 j}>0,(j=A, B, D, E)$. Considering the constraints $\quad \sum_{j=A, B, D, E} \lambda_{j}=1, \lambda_{j} \geq 0$, the constraint $\sum_{j=A, B, D, E} \lambda_{j} x_{2 j} \leq \theta x_{2 C}$ of the conventional input-oriented VRS superefficiency model [5] is not fulfilled. Thus, $\mathrm{DMU}_{C}$ 
TABLE 1: Example data and superefficiency results.

\begin{tabular}{|c|c|c|c|c|c|c|}
\hline \multirow{3}{*}{ DMU } & \multicolumn{3}{|c|}{ Original data } & \multirow{3}{*}{ Conventional input-oriented SE } & \multirow{3}{*}{ N-L SE } & \multirow{3}{*}{ New model (11) } \\
\hline & \multicolumn{2}{|c|}{ Input } & Output & & & \\
\hline & $x_{1}$ & $x_{2}$ & $y$ & & & \\
\hline A & 2 & 4 & 2 & 2.5000 & 2.5000 & 1.3000 \\
\hline B & 8 & 3 & 4 & 0.8132 & 0.8741 & 0.8994 \\
\hline $\mathrm{C}$ & 12 & 0 & 6 & Infeasible & Infeasible & 1.6000 \\
\hline $\mathrm{D}$ & 8 & 4 & 5 & 0.8500 & 0.9331 & 0.9333 \\
\hline $\mathrm{E}$ & 5 & 5 & 5 & 1.6000 & 1.2000 & 1.1500 \\
\hline
\end{tabular}

TABLE 2: Comparison of various VRS superefficiency models.

\begin{tabular}{|c|c|c|c|c|c|c|c|c|}
\hline DMU & Standard radial & $R$ & Beta $_{N-L}$ & N-L & $R$ & Beta $_{\text {NEW }}$ & New model (11) & $R$ \\
\hline NIPPON & 0.9888 & 17 & 0.0043 & 0.9957 & 17 & 0.0015 & 0.9985 & 17 \\
\hline CATHAY & 0.9209 & 20 & 0.0449 & 0.9551 & 20 & 0.0128 & 0.9872 & 18 \\
\hline GARUDA & 0.7419 & 28 & 0.1592 & 0.8408 & 28 & 0.0316 & 0.9684 & 24 \\
\hline JAL & 1.7463 & 3 & -0.2034 & 1.2034 & 3 & -0.1196 & 1.1196 & 1 \\
\hline MALAYSIA & 0.7741 & 27 & 0.1342 & 0.8658 & 27 & 0.0266 & 0.9734 & 23 \\
\hline QANTAS & 1.1330 & 9 & -0.0601 & 1.0601 & 9 & -0.0195 & 1.0195 & 9 \\
\hline SAUDIA & 1.1638 & 8 & -0.0798 & 1.0798 & 8 & -0.0165 & 1.0165 & 10 \\
\hline SINGAPORE & 1.4546 & 4 & -0.1707 & 1.1707 & 4 & -0.0616 & 1.0616 & 3 \\
\hline AUSTRIAN & 2.4309 & 2 & -1.4309 & 2.4309 & 1 & -0.0596 & 1.0596 & 4 \\
\hline BRITISH & 0.8915 & 22 & 0.0529 & 0.9471 & 22 & 0.0382 & 0.9618 & 27 \\
\hline FINNAIR & 1.3865 & 5 & -0.2075 & 1.2075 & 2 & -0.0206 & 1.0206 & 7 \\
\hline IBERIA & 0.7912 & 26 & 0.1131 & 0.8869 & 26 & 0.0374 & 0.9626 & 26 \\
\hline LUFTHANSA & Inf. & 1 & -0.0951 & 1.0951 & 6 & -0.0752 & 1.0752 & 2 \\
\hline SAS & 0.8740 & 24 & 0.0648 & 0.9352 & 24 & 0.0182 & 0.9818 & 21 \\
\hline SWISS & 1.0885 & 12 & -0.0454 & 1.0454 & 12 & -0.0129 & 1.0129 & 11 \\
\hline PORTUGAL & 1.2275 & 6 & -0.1108 & 1.1108 & 5 & -0.0120 & 1.0120 & 12 \\
\hline CANADA & 0.9252 & 19 & 0.0374 & 0.9626 & 19 & 0.0137 & 0.9863 & 19 \\
\hline AMER WEST & 1.1943 & 7 & -0.0913 & 1.0913 & 7 & -0.0201 & 1.0201 & 8 \\
\hline AMERICAN & Inf. & 10 & -0.0535 & 1.0535 & 10 & -0.0522 & 1.0522 & 5 \\
\hline CANADIAN & 0.9061 & 21 & 0.0469 & 0.9531 & 21 & 0.0147 & 0.9853 & 20 \\
\hline CONTINENTAL & 1.0015 & 16 & -0.0007 & 1.0007 & 16 & -0.0005 & 1.0005 & 16 \\
\hline DELTA & 0.9433 & 18 & 0.0287 & 0.9713 & 18 & 0.0250 & 0.9750 & 22 \\
\hline EASTERN & 0.8344 & 25 & 0.0880 & 0.9120 & 25 & 0.0338 & 0.9662 & 25 \\
\hline NORTHWEST & 1.0175 & 14 & -0.0079 & 1.0079 & 14 & -0.0064 & 1.0064 & 14 \\
\hline PANAM & 1.0290 & 13 & -0.0138 & 1.0138 & 13 & -0.0080 & 1.0080 & 13 \\
\hline TWA & 1.0088 & 15 & -0.0044 & 1.0044 & 15 & -0.0027 & 1.0027 & 15 \\
\hline UNITED & Inf. & 11 & -0.0515 & 1.0515 & 11 & -0.0515 & 1.0515 & 6 \\
\hline US AIR & 0.8752 & 23 & 0.0644 & 0.9356 & 23 & 0.0423 & 0.9577 & 28 \\
\hline
\end{tabular}

is infeasible in the conventional input-oriented VRS superefficiency model.

Column 6 of Table 1 presents the superefficiency scores obtained from the N-L model [14]. For $\mathrm{DMU}_{A}$, the N-L superefficiency score of $\mathrm{DMU}_{A}$ is 2.5000. Actually, $x_{1 A}=2$, $2 x_{1 A}<x_{1 j},(j=B, C, D, E)$ and $\sum_{j=B, C, D, E} \lambda_{j}=1, \lambda_{j} \geq 0$, thus $\sum_{j=B, C, D, E} \lambda_{j} x_{1 j} \geq 2 x_{1 A}$. The optimal objective function value $(\beta)$ is lower than -1 , and the output bundle $(1+\beta) y_{A}$ is negative, which is impossible. For $\mathrm{DMU}_{C}, x_{2 C}=0$, however, $x_{2 j}>0,(j=A, B, D, E)$. Considering the constraints $\sum_{j=A, B, D, E} \lambda_{j}=1, \lambda_{j} \geq 0$, the first constraint $\sum_{j=A, B, D, E} \lambda_{j} x_{2 j} \leq(1-\beta) x_{2 C}$ of the N-L superefficiency model (4) failed. Thus, $\mathrm{DMU}_{C}$ has the infeasible solution.

Column 7 of Table 1 exhibits the results obtained from our new model (11). Similar to the N-L superefficiency model, DMUs A, C, and E are superefficient with the negative optimal function value $(\beta)$. The proposed DDF- based VRS superefficiency model (11) in the current note can fully rank all DMUs.

\section{Application Examples}

In this section, we make comparisons between our first proposed DDF-based superefficiency under VRS and several previous superefficiency measures by example of the airline companies' dataset. The dataset is taken from Coelli et al. [23], which was also analyzed by Ray [14]. This sample is composed of 28 airline companies including three main regions in the world: Asia/Oceania, Europe, and North America. The four input variables are labor (pilots, crew, maintenance staff, etc), fuel, other inputs (e.g., various office and maintenance materials and services), and fixed capital. The two outputs are passenger (passenger-kilometres) and freight (tonne-kilometres of freight). Table 2 presents 
different efficiency results from different VRS superefficiency models.

Table 2 exhibits various superefficiency measures obtained from the standard input-oriented VRS model [5], N-L superefficiency model Ray [14], and our new DDF-based superefficiency model (11), respectively.

Columns 2 and 3 report the superefficiency and rankings obtained from the standard input-oriented VRS superefficiency model. JAL, QANTAS, SAUDIA, SINGAPORE, AUSTRIAN, FINNAIR, LUFTHANSA, SWISS, PORTUGAL, AMER WEST, AMERICAN, CONTINENTAL, NORTHWEST, PANAM, TWA, and UNITED are efficient firms. Among these efficient firms, except for three firms (LUFTHANSA, AMERICAN, and UNITED) which are infeasible in the standard input-oriented VRS superefficiency model, the superefficiency scores of the other firms exceed one.

Columns 4 and 5 show the directional distance function values $(\beta)$ and $\mathrm{N}$-L superefficiency scores $(1-\beta)$ [14], respectively. We obtain the same superefficient firms as the conventional VRS superefficiency model but with different superefficiency scores. From column 4 in Table 2, the directional distance function value $(\beta)$ of AUSTRIAN is -1.4309 , which means the output bundle $\left((1+\beta) y_{k}\right)$ is negative. The firm AUSTRIAN is also regarded as N-L strong superefficient (NLSSE) as its superefficiency score equals to 2.4309 which is greater than two [14].

Columns 7 and 8 display the directional distance function values $(\beta)$ and superefficiency scores obtained from our new DDF-based VRS superefficiency model (11), respectively. We also obtain the negative directional distance function values $(\beta)$ for the superefficient DMUs (JAL, QANTAS, SAUDIA, SINGAPORE, AUSTRIAN, FINNAIR, LUFTHANSA, SWISS, PORTUGAL, AMER WEST, AMERICAN, CONTINENTAL, NORTHWEST, PANAM, TWA, and UNITED) with the superefficiency scores greater than one. The new VRS superefficiency model based on the DDF can fully rank all DMUs (here refers to airline firms) with different efficiency scores.

\section{Conclusions}

In this paper, we focus on the DDF-based superefficiency models under VRS technology situation. We discuss the feasible conditions for the proper directional vector selection in the superefficiency measure to address the infeasibility issue and develop a new DDF-based VRS superefficiency model with a new and specified directional vector which differs from Chen et al. [7] and Lin and Chen [15]. In the new model, the infeasibility problems occurred in conventional VRS superefficiency and N-L superefficiency models cannot exist anymore. Furthermore, our proposed superefficiency measure does not need to predetermine the parameter as Chen et al.'s [7] and LR's modified superefficiency models [8]. Additionally, our new DDF-based VRS superefficiency model keeps unit-invariant when dealing with the measured input-output values. The results from numerical and application examples in the paper show that the proposed approach could fully rank all DMUs, and it does not confront the infeasibility. In real-world application, we suggest using the proposed method to give a fully ranking result for decision makers and to help selecting the best one or several top performers to make further managerial analysis.

\section{Data Availability}

Previous studies used to support this study are cited at relevant places within the text as references.

\section{Conflicts of Interest}

The authors declare that there are no conflicts of interest regarding the publication of this paper.

\section{Acknowledgments}

The authors would like to thank the China Postdoctoral Science Foundation (no. 2019M662210).

\section{References}

[1] A. Charnes, W. W. Cooper, and E. Rhodes, "Measuring the efficiency of decision making units," European Journal of Operational Research, vol. 2, no. 6, pp. 429-444, 1978.

[2] A. Emrouznejad and G.-l. Yang, "A survey and analysis of the first 40 years of scholarly literature in DEA: 1978-2016," SocioEconomic Planning Sciences, vol. 61, pp. 4-8, 2018.

[3] Y. Sun, H. Huang, and C. Zhou, "DEA game cross-efficiency model to urban public infrastructure investment comprehensive efficiency of China," Mathematical Problems in Engineering, vol. 2016, Article ID 9814313, 10 pages, 2016.

[4] A. Aldamak and S. Zolfaghari, "Review of efficiency ranking methods in data envelopment analysis," Measurement, vol. 106, pp. 161-172, 2017.

[5] P. Andersen and N. C. Petersen, "A procedure for ranking efficient units in data envelopment analysis," Management Science, vol. 39, no. 10, pp. 1261-1264, 1993.

[6] L. M. Seiford and J. Zhu, "Infeasibility of super-efficiency data envelopment analysis models," INFOR: Information Systems and Operational Research, vol. 37, no. 2, pp. 174-187, 1999.

[7] Y. Chen, J. Du, and J. Huo, "Super-efficiency based on a modified directional distance function," Omega, vol. 41, no. 3, pp. 621-625, 2013.

[8] C. A. K. Lovell and A. P. B. Rouse, "Equivalent standard DEA models to provide super-efficiency scores," Journal of the Operational Research Society, vol. 54, no. 1, pp. 101-108, 2003.

[9] Y. Chen, "Ranking efficient units in DEA," Omega, vol. 32, no. 3, pp. 213-219, 2004.

[10] Y. Chen, "Measuring super-efficiency in DEA in the presence of infeasibility," European Journal of Operational Research, vol. 161, no. 2, pp. 545-551, 2005.

[11] W. D. Cook and L. M. Seiford, "Data envelopment analysis (DEA)-thirty years on," European Journal of Operational Research, vol. 192, no. 1, pp. 1-17, 2009.

[12] R. G. Chambers, Y. Chung, and R. Färe, "Benefit and distance functions," Journal of Economic Theory, vol. 70, no. 2, pp. 407-419, 1996.

[13] F. Yang, F. Wei, Y. Li, Y. Huang, and Y. Chen, "Expected efficiency based on directional distance function in data envelopment analysis," Computers \& Industrial Engineering, vol. 125, pp. 33-45, 2018. 
[14] S. C. Ray, "The directional distance function and measurement of superefficiency: an application to airlines data," Journal of the Operational Research Society, vol. 59, no. 6, pp. 788-797, 2008.

[15] R. Lin and Z. Chen, "Super-efficiency measurement under variable return to scale: an approach based on a new directional distance function," Journal of the Operational Research Society, vol. 66, no. 9, pp. 1506-1510, 2015.

[16] M. C. A. S. Portela, E. Thanassoulis, and G. Simpson, "Negative data in DEA: a directional distance approach applied to bank branches," Journal of the Operational Research Society, vol. 55, no. 10, pp. 1111-1121, 2004.

[17] A. Hadi-Vencheh and A. Esmaeilzadeh, "A new superefficiency model in the presence of negative data," Journal of the Operational Research Society, vol. 64, no. 3, pp. 396-401, 2013.

[18] F. Wei, J. Song, C. Jiao, and F. Yang, "A modified slacks-based ranking method handling negative data in data envelopment analysis," Expert Systems, vol. 36, no. 1, Article ID e12329, 2019.

[19] R. D. Banker, A. Charnes, and W. W. Cooper, "Some models for estimating technical and scale inefficiencies in data envelopment analysis," Management Science, vol. 30, no. 9, pp. 1078-1092, 1984.

[20] R. G. Chambers, Y. Chung, and R. Färe, "Profit, directional distance functions, and Nerlovian efficiency," Journal of Optimization Theory and Applications, vol. 98, no. 2, pp. 351-364, 1998.

[21] W. W. Cooper, K. S. Park, and J. T. Pastor, "RAM: a range adjusted measure of inefficiency for use with additive models, and relations to other models and measures in DEA," Journal of Productivity analysis, vol. 11, no. 1, pp. 5-42, 1999.

[22] C. K. Lovell and J. T. Pastor, "Units invariant and translation invariant DEA models," Operations Research Letters, vol. 18, no. 3, pp. 147-151, 1995.

[23] T. Coelli, E. Grifell-Tatjé, and S. Perelman, "Capacity utilisation and profitability: a decomposition of short-run profit efficiency," International Journal of Production Economics, vol. 79, no. 3, pp. 261-278, 2002. 